\title{
Robust stability analysis of machining operations
}

\author{
David Hajdu • Tamas Insperger - Gabor Stepan
}

Published online: 20 April 2016

\begin{abstract}
Prediction of machine tool chatter requires the characterization of dynamic of the machine-toolworkpiece system by means of frequency response functions (FRFs). Uncertainties of the measured FRFs result in uncertainties of the calculated stability diagrams, therefore robustness of stability prediction against parameter perturbations is of high importance. Although there exist methods to determine robust stability in terms of stability radii, these methods either give a conservative estimate of the real uncertainties or are limited to perturbations of a few modal parameters, only. In this paper, a frequency-domain approach is presented to determine robust stability boundaries using the measured FRFs directly without any modal parameter identification. The method is based on an envelope fitting around the measured FRFs combined with some considerations of the single-frequency method. The application of the method is demonstrated in case of a turning operation, where the machine tool structure is characterized by a series of FRF measurements.
\end{abstract}

Keywords Robustness - Stability - Sensitivity · FRF . Chatter · Turning

\section{Introduction}

Reliable prediction of technological parameters without producing machine tool chatter is a highly important task for efficiency-oriented machine tool centers. After the pioneering work of Tobias [31] and Tlusty [30] in the 1950 s and $1960 \mathrm{~s}$, the so-called regenerative effect

D. Hajdu $(\varangle)$ · T. Insperger · G. Stepan

Department of Applied Mechanics

Budapest University of Technology and Economics

Budapest, Hungary

E-mail: hajdu@mm.bme.hu became the most commonly accepted explanation for machine tool chatter. The vibrations of the tool are copied onto the surface of the workpiece, which modifies the chip thickness and induces variation in the cutting-force one revolution later. From dynamic systems point of view, chatter is associated with the loss of stability of the steady-state (chatter-free) machining process followed by a large amplitude self-excited vibration between the tool and the workpiece usually involving intermittent loss of contact. Stability properties of machining processes are depicted by the so-called stability lobe diagrams, which plot the maximum stable depths of cut without producing chatter versus the spindle speed. These diagrams provide a guide to the machinist to select the optimal technological parameters in order to achieve maximum material removal rate without chatter.

Chatter prediction techniques can be classified into two groups: frequency-domain methods and time-domain methods [1]. Frequency-domain methods, such as the single frequency solution, the multi frequency solution $[2,4]$, the extended multi frequency solution [3], can apply the measured frequency response functions (FRFs) directly. Time-domain methods, such as the semi-discretization method [14,15], full-discretization method [6], Chebyshev collocation method $[5,32]$, spectral element method [17], the implicit subspace iteration method [36] or the integration method [37], just to mention a few, require fitted modal parameters as input.

In spite of the wide spectrum of the available efficient numerical techniques, the predicted stability lobe diagrams often do not match experimental cutting tests [29]. One reason for this can be identified in the limitations of models of machine tool chatter. Most models in the literature use linear systems, although nonlinear effects also influence global stability properties $[28,7]$. 
Another reason for the differences between predicted and measured stability lobe diagrams is the uncertainties of the measured FRFs. For frequency-domain methods, these uncertainties directly affect the generated stability lobe diagrams. For time-domain methods, the uncertainties of the FRF are manifested as uncertainties of the fitted modal parameters, which, again, affect stability lobe diagrams. In this case, the number of modes to be involved in the fitting and the properties of the mechanical model used for the fitting (e.g., proportional vs. non-proportional damping, symmetric vs. non-symmetric FRF matrix) also strongly affect the structure of the stability lobe diagrams $[22,37,11,10]$.

A dynamical system with an uncertain parameter $p$ bounded by the limits $p_{\min } \leq p \leq p_{\max }$ is called robustly stable if it is stable for any $p \in\left[p_{\min }, p_{\max }\right]$. For $N$ uncertain parameters $p_{i} \in\left[p_{i, \min }, p_{i, \max }\right], i=$ $1,2, \ldots, N$, the Edge Theorem combined with the Zero Exclusion method can be applied after determining the vertices corresponding to the limit values of $p_{i}[23,9,8]$.

A widely used approach in robust stability analysis is the so-called pseudospectra and stability radius method which gives the exact robust stability boundaries corresponding to bounded static perturbations. A detailed introduction to the concept of pseudospectra can be found in [33]. Stability radius for structured complex perturbations was presented in [12]. Real stability radius was introduced in [24]. An overview on pseudospectra and robust stability analysis for timedelay systems can be found in [20]. Real stability radius for time-delay systems was introduced in [21] and in [13]. The formulas for unstructured and weighted structured complex stability radii for time-delay systems were given in [19] and [35], respectively.

In this paper, the sensitivity of the stability charts of turning operations with respect to uncertain FRF and uncertain fitted modal parameters is studied. First, the effect of the uncertainties of the modal parameters are analyzed for time-domain techniques using the stability radii concept based on $[24,12,21,19]$. Then, inspired by the time-domain concept, a new frequency-domain estimation of the robust stability boundaries is proposed using the measured FRFs directly. Three different approaches are suggested based on different types of envelope of the FRFs. Finally, robust stability boundaries are determined for a turning operation with respect to the uncertainties of the measured FRFs.

\section{Robust stability analysis in time domain}

Time-domain chatter prediction requires fitted modal parameters. The accuracy of parameter fitting depends

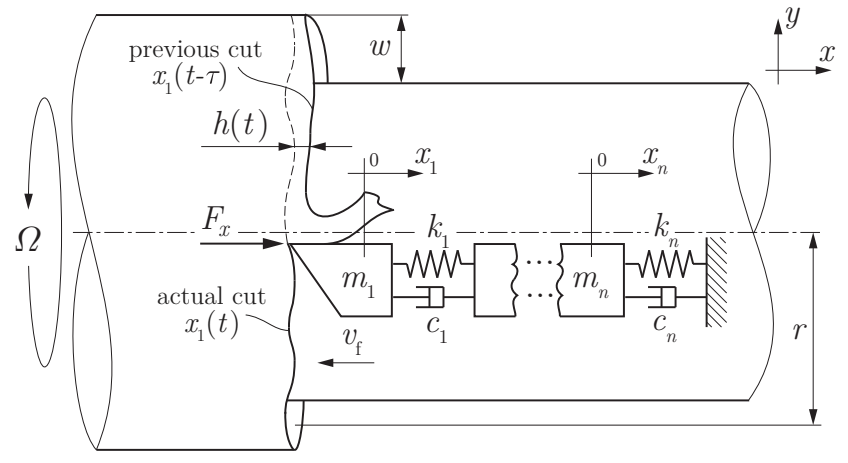

Fig. 1 Surface regeneration in an orthogonal cutting process with multiple vibration modes.

on many factors, such as the fitting algorithm, the number of degrees of freedom used in the model, and measurement precision. Some of the most widely used fitting algorithms are the Rational Fraction Polynomial method (RFP) [25], Least Squares Frequency-Domain algorithm (LSFD), Poly-reference Least Squares Complex Frequency-domain estimator, Frequency-domain Direct Parameter Identification (FDPI) method or Frequency-domain Maximum Likelihood Estimator (MLE) (see [34] and the references therein). Stability lobe diagrams can be constructed using the fitted modal parameters.

\subsection{Mechanical model of turning operation}

The dynamical model of an orthogonal cutting operation involving multiple vibration modes is shown in Fig. 1. The modes are projected to the direction $x$ of the surface regeneration. Note that vibrations in the $y$-direction do not affect linear stability properties [16]. An $n$-degrees-of-freedom mechanical model is used with generalized coordinates $\mathbf{x}(t)=\left(x_{1}(t), x_{2}(t), \ldots, x_{n}(t)\right)^{\mathrm{T}}$. The cutting-force acting on the tool tip according to the power law $[18,27]$ can be given as

$F_{x}(t)=K_{x} w h^{q}(t)$

where $K_{x}$ is the cutting-force coefficient in direction $x$, $w$ is the depth of cut, $h(t)$ is the instantaneous chip thickness and $q$ is the cutting-force exponent. Due to the vibrations of the tool, the chip thickness is determined by the feed motion, the current tool position and the previous position of the tool one revolution before. For constant spindle speeds, the regenerative time delay can be given explicitly as $\tau=60 / \Omega$, where $\Omega$ is the workpiece revolution given in $\mathrm{rpm}$. The instantaneous chip thickness can be calculated as

$h(t)=v_{\mathrm{f}} \tau+x_{1}(t-\tau)-x_{1}(t)$, 
where $v_{\mathrm{f}}$ is the feed velocity and $x_{1}$ indicates the position of the tool tip in direction $x$. The forcing vector $\mathbf{f}(t) \in \mathbb{R}^{n}$ is given as

$\mathbf{f}(t)=\left(\begin{array}{c}K_{x} w\left(v_{\mathrm{f}} \tau+x_{1}(t-\tau)-x_{1}(t)\right)^{q} \\ 0 \\ \vdots \\ 0\end{array}\right)$.

Local stability of the steady-state (chatter-free) motion can be analyzed by means of the linearized system. The general solution can be given as $\mathbf{x}(t)=\mathbf{x}_{\mathrm{s}}+\boldsymbol{\xi}(t)$, where $\mathbf{x}_{\mathbf{S}} \in \mathbb{R}^{n}$ is related to the static deformation and $\boldsymbol{\xi}(t) \in \mathbb{R}^{n}$ is a small perturbation around the equilibrium $\mathbf{x}(t) \equiv \mathbf{x}_{\mathbf{s}}$. After the linearization, the variational system is given by

$\mathbf{M} \ddot{\boldsymbol{\xi}}(t)+\mathbf{C} \dot{\boldsymbol{\xi}}(t)+\mathbf{K} \boldsymbol{\xi}(t)=\boldsymbol{\kappa}(\boldsymbol{\xi}(t-\tau)-\boldsymbol{\xi}(t))$,

where $\mathbf{M} \in \mathbb{R}^{n \times n}$ is the mass matrix, $\mathbf{C} \in \mathbb{R}^{n \times n}$ is the damping matrix, $\mathbf{K} \in \mathbb{R}^{n \times n}$ is the stiffness matrix and

$\boldsymbol{\kappa}=\left(\begin{array}{cccc}\kappa & 0 & \ldots & 0 \\ 0 & 0 & \ldots & 0 \\ \vdots & \vdots & & \vdots \\ 0 & 0 & \ldots & 0\end{array}\right) \in \mathbb{R}^{n \times n}$

with $\kappa=K_{x} w q\left(v_{\mathrm{f}} \tau\right)^{q-1}$ being the specific cutting-force coefficient. Note that $\kappa$ is linearly proportional to the depth of cut $w$, which is one of the main technological parameters.

Modal parameters are to be estimated using the measured FRF of the machine-tool-workpiece system. Uncertainties of the FRF generate uncertainties in the fitted modal parameters, which result in uncertainties in the system matrices $\mathbf{M}, \mathbf{C}$ and $\mathbf{K}$. The region of robust stability of Eq. (4) against parameter uncertainties can be determined using the concept of pseudospectra and stability radius.

\subsection{Pseudospectra and stability radius}

The $\epsilon$-pseudospectrum $\sigma_{\epsilon}$ of a matrix $\mathbf{A}$ is defined as

$\sigma_{\epsilon}:=\{\lambda \in \mathbb{C}: \lambda \in \sigma(\mathbf{A}+\delta \mathbf{A})$, where $\|\delta \mathbf{A}\|<\epsilon\}$,

where $\sigma$ denotes the spectrum, $\|\cdot\|$ is an arbitrary matrix norm and $\delta \mathbf{A}$ is a perturbation matrix [19]. It is known, that Eq. (6) is equivalent to

$\sigma_{\epsilon}:=\{\lambda \in \mathbb{C}:\|\mathrm{R}(\lambda, \mathbf{A})\|>1 / \epsilon\}$,

where $\mathrm{R}(\lambda, \mathbf{A})=(\lambda \mathbf{I}-\mathbf{A})^{-1}$ denotes the corresponding resolvent operator with $\mathbf{I}$ being the identity matrix
[33]. The $\epsilon$-pseudospectrum plays an important role in the definition of stability radius of time-delay systems.

In the next subsections, four concepts for the stability radius are described. First, in section 2.2.1, the complex stability radius is considered, where one of the system matrices $\mathbf{M}, \mathbf{C}$ or $\mathbf{K}$ is perturbed by complex values. In section 2.2.2, combined but still complex-valued perturbation of all the system matrices is given, which is called weighted complex stability radius. Since physical interpretation of complex-valued perturbations of systems with real parameters is controversial, two concepts of real stability radii are also given. In section 2.2 .3 and 2.2.4, real and weighted real stability radii are given.

\subsubsection{Complex stability radius}

Perturbations of the mass, damping and stiffness matrices can be described by the perturbation matrices $\delta \mathbf{M}, \delta \mathbf{C}$ and $\delta \mathbf{K}$, respectively. The perturbed equation in case of perturbation of the mass matrix $\mathbf{M}$ reads

$$
(\mathbf{M}+\delta \mathbf{M}) \ddot{\boldsymbol{\xi}}(t)+\mathbf{C} \dot{\boldsymbol{\xi}}(t)+\mathbf{K} \boldsymbol{\xi}(t)=\boldsymbol{\kappa}(\boldsymbol{\xi}(t-\tau)-\boldsymbol{\xi}(t)) .
$$

According to the stability radii theorem (see [20]), the unstructured complex stability radius of the system corresponding to complex-valued perturbations of the mass matrix $\mathbf{M}$ can be calculated as

$r_{\mathbb{C}}^{\mathbf{M}}=\left(\sup _{\omega \geq 0}\left\|(\mathrm{i} \omega)^{2}(\mathbf{D}(\omega))^{-1}\right\|_{2}\right)^{-1}$,

where

$\mathbf{D}(\omega)=(\mathrm{i} \omega)^{2} \mathbf{M}+\mathrm{i} \omega \mathbf{C}+\mathbf{K}+\boldsymbol{\kappa}\left(1-\mathrm{e}^{-\mathrm{i} \omega \tau}\right)$

is the characteristic matrix evaluated on the imaginary axis and $\|\cdot\|_{2}$ denotes the spectral norm. If the nominal system with $\delta \mathbf{M}=\mathbf{0}$ is stable, then the perturbed system with any $\delta \mathbf{M}$ satisfying the condition $\|\delta \mathbf{M}\|_{2}<r_{\mathbb{C}}^{\mathbf{M}}$ is stable, too. Consequently, the robust stability boundaries for perturbations of different sizes are given by the contour curves of $r_{\mathbb{C}}^{\mathbf{M}}$.

In case of perturbations of the damping matrix as $\mathbf{C}+\delta \mathbf{C}$ or the stiffness matrix as $\mathbf{K}+\delta \mathbf{K}$, complex stability radius can be given in a similar manner as

$r_{\mathbb{C}}^{\mathbf{C}}=\left(\sup _{\omega \geq 0}\left\|\mathrm{i} \omega(\mathbf{D}(\omega))^{-1}\right\|_{2}\right)^{-1}$,

or

$r_{\mathbb{C}}^{\mathbf{K}}=\left(\sup _{\omega \geq 0}\left\|(\mathbf{D}(\omega))^{-1}\right\|_{2}\right)^{-1}$,

respectively. 
The stability radii $r_{\mathbb{C}}^{\mathbf{M}}, r_{\mathbb{C}}^{\mathbf{C}}$ and $r_{\mathbb{C}}^{\mathbf{K}}$ allow the perturbation only in one of the system matrices, in $\mathbf{M}$, in $\mathbf{C}$, or in $\mathbf{K}$, respectively. The case when all the three matrices are perturbed at the same time can be handled by the weighted stability radius.

\subsubsection{Weighted complex stability radius}

The perturbed equation for the case when all the system matrices are perturbed to different extent reads

$$
\begin{aligned}
&(\mathbf{M}+\delta \mathbf{M}) \ddot{\boldsymbol{\xi}}(t)+(\mathbf{C}+\delta \mathbf{C}) \dot{\boldsymbol{\xi}}(t)+ \\
&(\mathbf{K}+\delta \mathbf{K}) \boldsymbol{\xi}(t)=\boldsymbol{\kappa}(\boldsymbol{\xi}(t-\tau)-\boldsymbol{\xi}(t)) .
\end{aligned}
$$

This type of combined perturbation can be described by the perturbation matrix of the form

$\boldsymbol{\Delta}:=\left(\begin{array}{c}w_{\mathrm{M}} \delta \mathbf{M} \\ w_{\mathrm{C}} \delta \mathbf{C} \\ w_{\mathrm{K}} \delta \mathbf{K}\end{array}\right)$

where $w_{\mathrm{M}}, w_{\mathrm{C}}$ and $w_{\mathrm{K}}$ are the weights of the perturbation of the mass, damping and stiffness matrices, respectively. If $\|\delta \mathbf{M}\|<\varepsilon_{\mathrm{M}}\|\mathbf{M}\|,\|\delta \mathbf{C}\|<\varepsilon_{\mathrm{C}}\|\mathbf{C}\|$ and $\|\delta \mathbf{K}\|<\varepsilon_{\mathrm{K}}\|\mathbf{K}\|$, where $\varepsilon_{\mathrm{M}}, \varepsilon_{\mathrm{C}}$ and $\varepsilon_{\mathrm{K}}$ are the radii of uncertainties, then the corresponding weights are

$w_{\mathrm{M}}=\frac{1}{\varepsilon_{\mathrm{M}}\|\mathbf{M}\|}, \quad w_{\mathrm{C}}=\frac{1}{\varepsilon_{\mathrm{C}}\|\mathbf{C}\|}, \quad w_{\mathrm{K}}=\frac{1}{\varepsilon_{\mathrm{K}}\|\mathbf{K}\|}$.

For instance, if mass matrix $\mathbf{M}$ is perturbed by maximum $2 \%$, then $w_{\mathrm{M}}=(0.02\|\mathbf{M}\|)^{-1}$. If $w_{\mathrm{M}} \rightarrow \infty$, then no perturbation on the mass matrix $\mathbf{M}$ is allowed [19]. This formalism allows the perturbations satisfying

$\left(\|\boldsymbol{\Delta}\|_{2}\right)^{2}=\left(\frac{\|\delta \mathbf{M}\|}{\varepsilon_{\mathrm{M}}\|\mathbf{M}\|}\right)^{2}+\left(\frac{\|\delta \mathbf{C}\|}{\varepsilon_{\mathrm{C}}\|\mathbf{C}\|}\right)^{2}+\left(\frac{\|\delta \mathbf{K}\|}{\varepsilon_{\mathrm{K}}\|\mathbf{K}\|}\right)^{2} \leq 1$.

Thus, the allowed perturbations lie within an ellipsoid of main axes $\varepsilon_{\mathrm{M}}\|\mathbf{M}\|, \varepsilon_{\mathrm{C}}\|\mathbf{C}\|$ and $\varepsilon_{\mathrm{k}}\|\mathbf{K}\|$ in the parameter space $(\|\delta \mathbf{M}\|,\|\delta \mathbf{C}\|,\|\delta \mathbf{K}\|)$.

According to [19], the complex stability radius corresponding to the perturbation matrix $\Delta$ can be calculated as

$r_{\mathbb{C}}^{\Delta}=\left(\sup _{\omega \geq 0}\left\|(\mathbf{D}(\omega))^{-1} \mathbf{w}(\mathrm{i} \omega)\right\|_{2}\right)^{-1}$,

where

$\mathbf{w}(\mathrm{i} \omega)=\left(\frac{(\mathrm{i} \omega)^{2}}{w_{\mathrm{M}}} \mathbf{I} \quad \frac{(\mathrm{i} \omega)}{w_{\mathrm{C}}} \mathbf{I} \frac{1}{w_{\mathrm{K}}} \mathbf{I}\right) \in \mathbb{C}^{n \times 3 n}$

is the complex weight function with $\mathbf{I}$ being the $n \times n$ identity matrix. If the nominal system is stable and $r_{\mathbb{C}}^{\Delta}<1$, then the system is robustly stable for any perturbations satisfying $\|\boldsymbol{\Delta}\|_{2}<1$. Consequently the robust stability boundary associated with the uncertainty radii $\varepsilon_{\mathrm{M}}, \varepsilon_{\mathrm{C}}$ and $\varepsilon_{\mathrm{K}}$ is given by the contour curve $r_{\mathbb{C}}^{\Delta}=1$. Contour curves $r_{\mathbb{C}}^{\Delta}=z$ with any $z \in \mathbb{R}^{+}$give the robust stability boundaries associated with the uncertainty radii $z \varepsilon_{\mathrm{M}}, z \varepsilon_{\mathrm{C}}$ and $z \varepsilon_{\mathrm{K}}$.

The stability radii $r_{\mathbb{C}}^{\mathbf{M}}, r_{\mathbb{C}}^{\mathbf{C}}, r_{\mathbb{C}}^{\mathbf{K}}$ and $r_{\mathbb{C}}^{\Delta}$ correspond to the complex-valued perturbation of the matrices $\mathbf{M}$, $\mathbf{C}$ and $\mathbf{K}$ and provide therefore a conservative estimation of the true stability radius. Actual robust stability properties are better described by real stability radii.

\subsubsection{Real stability radius}

If only real entries of the perturbation matrices $\delta \mathbf{M}$, $\delta \mathbf{C}$ and $\delta \mathbf{K}$ are allowed, then the corresponding real stability radius can be calculated following [21] and [13] as

$$
\begin{aligned}
r_{\mathbb{R}}^{\mathbf{M}} & =\left(\sup _{\omega \geq 0} \mu_{\mathbb{R}}\left((\mathrm{i} \omega)^{2}(\mathbf{D}(\omega))^{-1}\right)\right)^{-1}, \\
r_{\mathbb{R}}^{\mathbf{C}} & =\left(\sup _{\omega \geq 0} \mu_{\mathbb{R}}\left(\mathrm{i} \omega(\mathbf{D}(\omega))^{-1}\right)\right)^{-1} \\
r_{\mathbb{R}}^{\mathbf{K}} & =\left(\sup _{\omega \geq 0} \mu_{\mathbb{R}}\left((\mathbf{D}(\omega))^{-1}\right)\right)^{-1}
\end{aligned}
$$

where

$\mu_{\mathbb{R}}(\mathbf{W}(\omega))=\inf _{\gamma \in(0,1]} \sigma_{2}\left(\begin{array}{cc}\mathfrak{R e}(\mathbf{W}(\omega)) & -\gamma \mathfrak{I m}(\mathbf{W}(\omega)) \\ \gamma^{-1} \mathfrak{I m}(\mathbf{W}(\omega)) & \mathfrak{R e}(\mathbf{W}(\omega))\end{array}\right)$.

for an arbitrary matrix function $\mathbf{W}(\omega)$. Here $\sigma_{2}$ denotes the second largest singular value. Note, that Eq. (22) is a unimodal function in $\gamma$, therefore a golden section method can be applied to find its minimum [24].

\subsubsection{Weighted real stability radius}

Weighted real stability radius is a straightforward combination of unweighted real $[21,13]$ and weighted complex $[19,35]$ stability radii and can be calculated as

$r_{\mathbb{R}}^{\Delta}=\left(\sup _{\omega \geq 0} \mu_{\mathbb{R}}\left((\mathbf{D}(\omega))^{-1} \mathbf{w}(\omega)\right)\right)^{-1}$

where $\boldsymbol{\Delta}$ and $\mathbf{w}(\omega)$ are defined as in (14) and in (18), respectively. If the nominal system is stable and $r_{\mathbb{R}}^{\boldsymbol{\Delta}}<1$, then the system is robustly stable for any real-valued perturbations satisfying $\|\boldsymbol{\Delta}\|_{2}<1$. Similarly to the previous cases, the robust stability boundaries associated with the uncertainty radii $\varepsilon_{\mathrm{M}}, \varepsilon_{\mathrm{C}}$, and $\varepsilon_{\mathrm{K}}$ are given by the contour curve $r_{\mathbb{R}}^{\Delta}=1$, while the contour curves $r_{\mathbb{R}}^{\Delta}=z, z \in \mathbb{R}^{+}$give the boundaries associated with the uncertainty radii $z \varepsilon_{\mathrm{M}}, z \varepsilon_{\mathrm{C}}$, and $z \varepsilon_{\mathrm{K}}$. 
Note, that the robust stability boundaries calculated using $r_{\mathbb{R}}^{\Delta}$ are still conservative since the perturbation measures give limit only on the norm of the perturbed matrices and do not consider the structure of the matrices. The condition can be sharpened using structured perturbations according to $[12,35]$. The method of structured perturbations for multiple-degrees-of-freedom systems is however limited since only certain number of combinations of the entries of the matrices can be perturbed individually.

\subsection{Analysis of a single-degree-of-freedom system}

In case of a single-degree-of-freedom system governed by

$$
m \ddot{\xi}(t)+c \dot{\xi}(t)+k \xi(t)=\kappa(\xi(t-\tau)-\xi(t)),
$$

the weighted real stability radius gives the exact robust stability boundaries for any combined perturbations of the modal mass $m$, damping $c$ and stiffness $k$. The corresponding characteristic equation on the imaginary axis is

$D(\omega)=m(\mathrm{i} \omega)^{2}+c \mathrm{i} \omega+k+\kappa-\kappa \mathrm{e}^{-\mathrm{i} \omega \tau}$.

In case of weighted perturbations of the system parameters as $m \rightarrow m+\delta m, c \rightarrow c+\delta c$ and $k \rightarrow k+\delta k$, the perturbation matrix reads

$\boldsymbol{\Delta}=\left(\begin{array}{c}w_{m} \delta m \\ w_{c} \delta c \\ w_{k} \delta k\end{array}\right)$

where $w_{m}, w_{c}$ and $w_{k}$ are the perturbation weights. If $|\delta m|<\varepsilon_{m}|m|,|\delta c|<\varepsilon_{c}|c|$ and $|\delta k|<\varepsilon_{k}|k|$, where $\varepsilon_{m}, \varepsilon_{c}$ and $\varepsilon_{k}$ are the radii of uncertainties, then the corresponding weights are

$w_{m}=\frac{1}{\varepsilon_{m} m}, \quad w_{c}=\frac{1}{\varepsilon_{c} c}, \quad w_{k}=\frac{1}{\varepsilon_{k} k}$.

Similarly to (16), the allowed perturbations lie within an ellipsoid defined by

$\left(\|\boldsymbol{\Delta}\|_{2}\right)^{2}=\left(\frac{\delta m}{\varepsilon_{m} m}\right)^{2}+\left(\frac{\delta c}{\varepsilon_{c} c}\right)^{2}+\left(\frac{\delta k}{\varepsilon_{k} k}\right)^{2} \leq 1$.

In this numerical study, the following nominal parameters were assumed: $m=1[\mathrm{~kg}], c=100[\mathrm{Ns} / \mathrm{m}]$, $k=10^{6}[\mathrm{~N} / \mathrm{m}]$. The results are presented for three different cases. Stability diagrams are constructed by evaluating Eq. (23) on a $400 \times 200$ grid of the parameter plane $(\Omega, \kappa)$. The frequency parameter $\omega$ was swept from 0 to $3000[\mathrm{rad} / \mathrm{s}]$. Parameter $\gamma$ was determined by

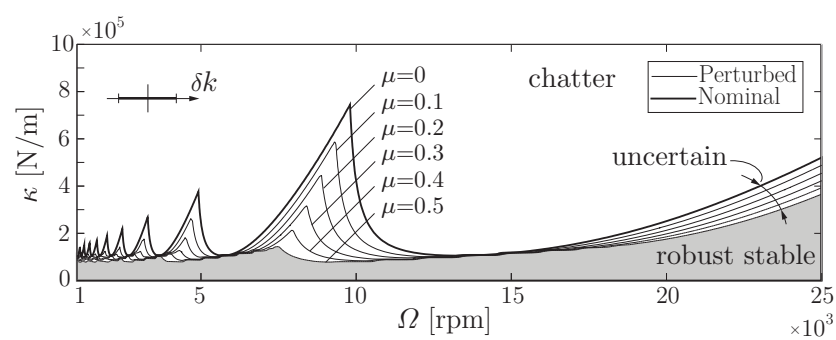

Fig. 2 Robust stability boundaries of Eq. (24) for uncertainty radii $\varepsilon_{m}=0, \varepsilon_{c}=0, \varepsilon_{k}=\mu$.

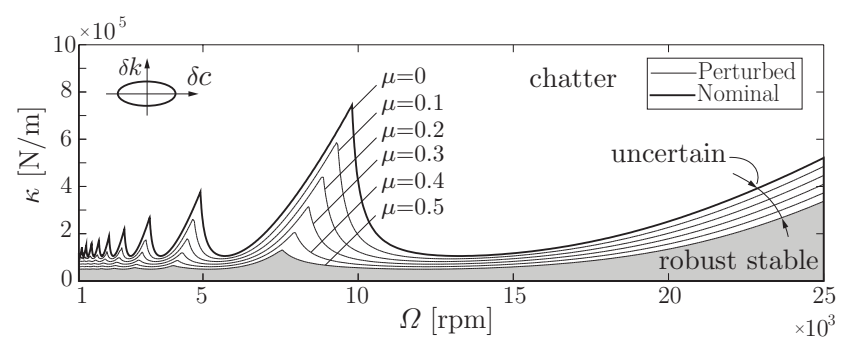

Fig. 3 Robust stability boundaries of Eq. (24) for uncertainty radii $\varepsilon_{m}=0, \varepsilon_{c}=\mu, \varepsilon_{k}=\mu$.

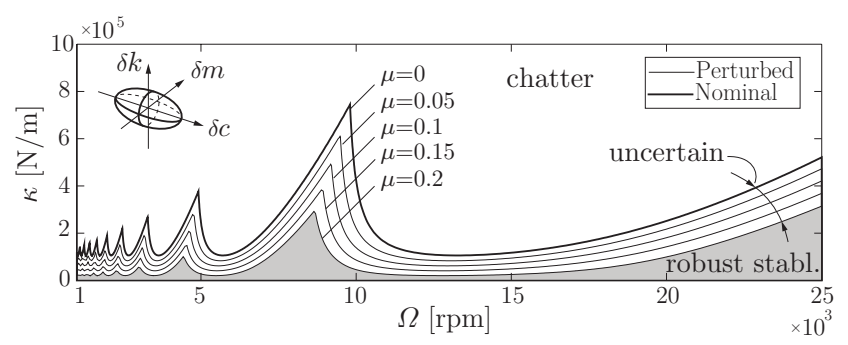

Fig. 4 Robust stability boundaries of Eq. (24) for uncertainty radii $\varepsilon_{m}=\mu, \varepsilon_{c}=4 \mu, \varepsilon_{k}=\mu$.

the golden section method [24]. The stability boundaries without perturbation is obtained by the contour level $r_{\mathbb{R}}^{\Delta}=0$.

In Fig. 2, only the stiffness $k$ is perturbed such that $|\delta k|<\varepsilon_{k}|k|$ with uncertainty radius $\varepsilon_{k}=\mu=$ $0,0.1, \ldots, 0.5$ and the mass and the damping parameters are fixed $\left(\varepsilon_{m}=\varepsilon_{c}=0\right)$. The corresponding weights are therefore $w_{m}=\infty, w_{c}=\infty, w_{k}=\left(\varepsilon_{k} k\right)^{-1}$. For instance, the case $\mu=0.1$ represents a maximum $\pm 10 \%$ perturbation of the stiffness parameter.

In Fig. 3, both the damping and the stiffness parameters are perturbed with the same uncertainty radii $\varepsilon_{c}=\varepsilon_{k}=\mu$, while the mass is fixed $\left(\varepsilon_{m}=0\right)$. Here, the case $\mu=0.1$ represents a maximum $\pm 10 \%$ perturbation of the stiffness parameter and a maximum $\pm 10 \%$ perturbation of the damping parameter.

Figure 4 shows the case when all of the three modal parameters are perturbed with uncertainty radii $\varepsilon_{m}=$ $\varepsilon_{k}=\mu$ and $\varepsilon_{c}=4 \mu$. Here, the case $\mu=0.1$ represents a maximum $\pm 10 \%$ perturbation of the stiffness param- 
eter, a maximum $\pm 40 \%$ perturbation of the damping parameter and a maximum $\pm 10 \%$ perturbation of the mass. It can be seen that perturbation of different system parameters gives different robust stability boundaries. For given perturbation bounds, the robust stability boundaries can exactly be determined.

Although the weighted real stability radius can easily be determined for single-degree-of-freedom systems, its application to multiple-degrees-of-freedom systems is limited since individual perturbations of the elements of the mass, damping and stiffness matrices cannot be described by this technique. In that case only conservative estimations can be obtained by giving conditions for the norm of the perturbation matrices. Another difficulty is that the calculation time of the second largest singular value increases with the number of perturbed parameters.

\section{Robust stability analysis in frequency domain}

In this section, a new method is presented to the robust stability analysis using the uncertainties of the measured FRF directly (see Fig. 5).

\subsection{Single-frequency method}

In this subsection the main steps of the single-frequency method is summarized for the stability analysis of systems with fixed parameters [1]. The definition of the FRF matrix $\mathbf{H}(\omega)$ gives

$\mathbf{H}(\omega) \mathbf{F}(\omega)=\mathbf{X}(\omega)$,

where $\mathbf{F}(\omega)=\mathscr{F}(\mathbf{f}(t)), \mathbf{X}(\omega)=\mathscr{F}(\boldsymbol{\xi}(t))$ and $\mathscr{F}$ denotes the Fourier transform. The Fourier transform of the parametric forcing $\mathbf{f}(t)=\boldsymbol{\kappa}(\boldsymbol{\xi}(t-\tau)-\boldsymbol{\xi}(t))$, which is the right hand side of Eq. (4), is

$\mathbf{F}(\omega)=\kappa\left(\mathrm{e}^{-\mathrm{i} \omega \tau}-1\right) \mathbf{X}(\omega)$.
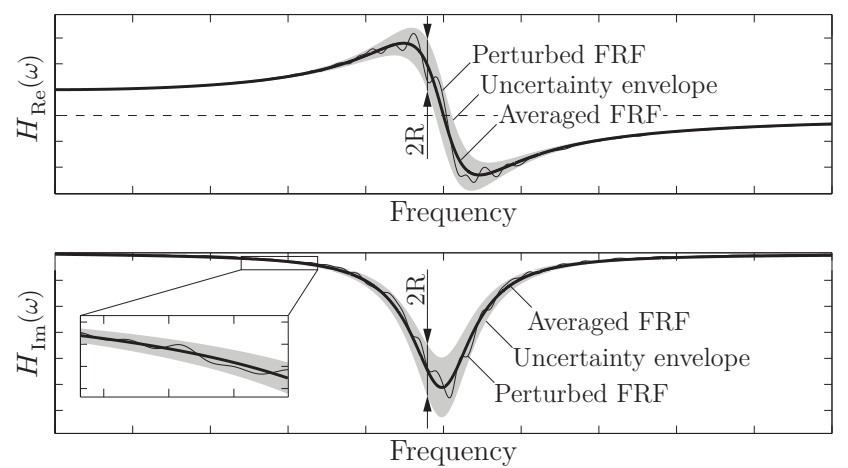

Fig. 5 Frequency response function of a single-degree-offreedom system with uncertainty.
Substitution of Eq. (29) into Eq. (30), and simplification yield

$\left(\mathbf{I}-\left(\mathrm{e}^{-\mathrm{i} \omega \tau}-1\right) \boldsymbol{\kappa} \mathbf{H}(\omega)\right) \mathbf{F}(\omega)=\mathbf{0}$.

The existence of the nontrivial solution implies

$\operatorname{det}\left(\mathbf{I}-\left(\mathrm{e}^{-\mathrm{i} \omega \tau}-1\right) \boldsymbol{\kappa} \mathbf{H}(\omega)\right)=0$,

which, considering the structure of $\kappa$ in (5), can be expressed as

$\mathrm{D}(\omega)=1-\left(\mathrm{e}^{-\mathrm{i} \omega \tau}-1\right) \kappa H_{11}(\omega)=0$,

where $H_{11}(\omega)$ is the measured tip-to-tip FRF (corresponding to the vibrations of the tool tip due to forcing at the tool tip). Note that Eq. (33) is the characteristic equation of the system evaluated on the imaginary axis. For the sake of simplicity, the notation $H(\omega):=H_{11}(\omega)$ is to be used in the sequel. If the inverse FRF is written as $H^{-1}(\omega)=\Lambda_{\operatorname{Re}}+\mathrm{i} \Lambda_{\mathrm{Im}}$, then the analytic solution for the transition curves, where the real parts of some characteristic roots are zero, can be given as

$\Omega=\frac{30 \omega}{\operatorname{arctg}\left(\frac{\Lambda_{\mathrm{Re}}}{\Lambda_{\mathrm{Im}}}\right)+j \pi}, \quad \kappa=-\frac{\Lambda_{\mathrm{Re}}^{2}+\Lambda_{\mathrm{Im}}^{2}}{2 \Lambda_{\mathrm{Re}}}$,

where $j=0,1,2 \ldots$ and $\omega \in[0, \infty)$. These transition curves separate the parameter plane into regions where the number of unstable characteristic roots is constant. Stable domains are the ones associated with zero unstable characteristic roots, which are the domains below the transition curves. In general, the number of unstable roots can be determined using the Stepan formulas [26].

\subsection{Robust stability approach}

The FRFs obtained from measurements are always loaded by noise and uncertainties, which can be represented as an uncertain envelope around the averaged FRF (see Fig. 5). Consequently, uncertainties of the FRF can be represented as

$H(\omega)=\bar{H}(\omega)+\delta H(\omega)$,

where $\bar{H}(\omega)$ is the averaged FRF and $\delta H(\omega)$ is the perturbation. Substitution of Eq. (35) into the characteristic equation (33) and separation to real and imaginary parts give

$\delta H_{\operatorname{Re}}(\omega)=-\frac{1}{2 \kappa}-\bar{H}_{\operatorname{Re}}(\omega)$,
$\delta H_{\operatorname{Im}}(\omega)=-\frac{\sin (\omega \tau)}{2 \kappa(1-\cos (\omega \tau))}-\bar{H}_{\operatorname{Im}}(\omega)$. 

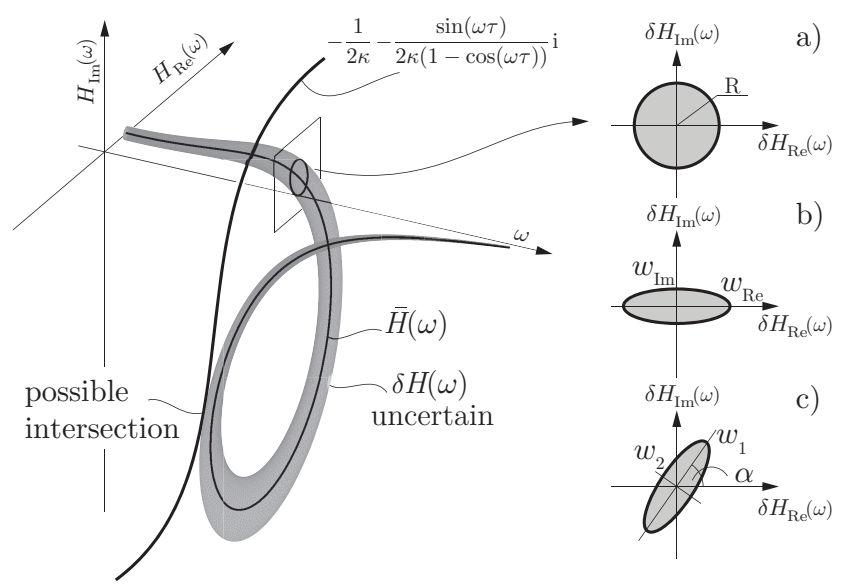

Fig. 6 Visualization of the solution of Eq. (36)-(37) for different types of perturbation envelopes: circular (a); elliptic (b); and rotated elliptic (c).

If there is a perturbation $\delta H(\omega)=\delta H_{\operatorname{Re}}(\omega)+\mathrm{i} \delta H_{\operatorname{Im}}(\omega)$, which satisfies Eqs. (36)-(37) for some $\omega$, then the system is not robustly stable. If Eqs. (36)-(37) cannot be satisfied by the possible/allowed perturbations for any $\omega$, then the system is robustly stable or robustly unstable depending on whether the averaged system is stable or unstable.

A bound for the uncertainties of the FRF can be given by an envelope about the averaged FRF. A possible envelope is a tube obtained by centering discs of radius $R(\omega)$ at $\bar{H}(\omega)$ (see Fig. 6a). In this case, a safety factor can be defined as

$S F_{\mathrm{a}}:=\min _{\omega \in[0, \infty)} \frac{\left|\delta H_{\mathrm{Re}}(\omega)+\mathrm{i} \delta H_{\operatorname{Im}}(\omega)\right|}{R(\omega)}$,

where $\delta H_{\operatorname{Re}}(\omega)$ and $\delta H_{\operatorname{Im}}(\omega)$ are provided by Eqs. (36) and (37), respectively. If $S F_{\mathrm{a}}<1$ then the system is not robustly stable, i.e., there exists a perturbation $\mid \delta H(\omega \mid) \leq R(\omega)$, for which the system is unstable. If $S F_{\mathrm{a}}>1$ then the system is robustly stable if the averaged model is stable or robustly unstable if the averaged model is unstable. The robust stability boundary is given by the contour curves $S F_{\mathrm{a}}=1$.

The accuracy of the robust stability analysis depends on the estimation of the envelope of the actual measured FRF. Circular approximation of the uncertainties of the FRF assumes the same additive perturbation in the real and the imaginary parts (see Fig. $6 a)$, which may overestimate the perturbations. A less conservative estimate is obtained if the real and the imaginary parts are perturbed to different extents such that

$$
\left(\frac{\delta H_{\operatorname{Re}}(\omega)}{w_{\operatorname{Re}}(\omega)}\right)^{2}+\left(\frac{\delta H_{\operatorname{Im}}(\omega)}{w_{\operatorname{Im}}(\omega)}\right)^{2} \leq 1
$$

(see Fig. $6 \mathrm{~b}$ ). Here $w_{\text {Re }}$ and $w_{\text {Im }}$ are the halves of the axes of the ellipse, which bounds the perturbations. In this case, the safety factor can be defined as

$$
S F_{\mathrm{b}}=\min _{\omega \in[0, \infty)} \sqrt{\left(\frac{\delta H_{\operatorname{Re}}(\omega)}{w_{\operatorname{Re}}(\omega)}\right)^{2}+\left(\frac{\delta H_{\operatorname{Im}}(\omega)}{w_{\operatorname{Im}}(\omega)}\right)^{2}} .
$$

An even better estimation of the envelope is a rotated ellipse characterized by the half-lengths $w_{1}(\omega)$ and $w_{2}(\omega)$ of its major and minor axes and by a rotation angle $\alpha(\omega)$ (see Fig. 6c). In this case, the perturbations satisfy

$$
\begin{aligned}
& \left(\frac{\delta H_{\mathrm{Re}} \cos (\alpha)+\delta H_{\operatorname{Im}} \sin (\alpha)}{w_{1}}\right)^{2} \\
& \quad+\left(\frac{\delta H_{\mathrm{Im}} \cos (\alpha)-\delta H_{\mathrm{Re}} \sin (\alpha)}{w_{2}}\right)^{2} \leq 1
\end{aligned}
$$

where the dependence on $\omega$ is not presented for the sake of brevity. The corresponding safety factor is

$$
\begin{aligned}
S F_{\mathrm{c}}= & \min _{\omega \in[0, \infty)}\left(\left(\frac{\delta H_{\mathrm{Re}} \cos (\alpha)+\delta H_{\mathrm{Im}} \sin (\alpha)}{w_{1}}\right)^{2}\right. \\
& \left.+\left(\frac{\delta H_{\mathrm{Im}} \cos (\alpha)-\delta H_{\mathrm{Re}} \sin (\alpha)}{w_{2}}\right)^{2}\right)^{1 / 2} .
\end{aligned}
$$

For all the above envelope types, robust stability boundaries are given by the contour curves $S F_{\mathrm{a}, \mathrm{b}, \mathrm{c}}=1$.
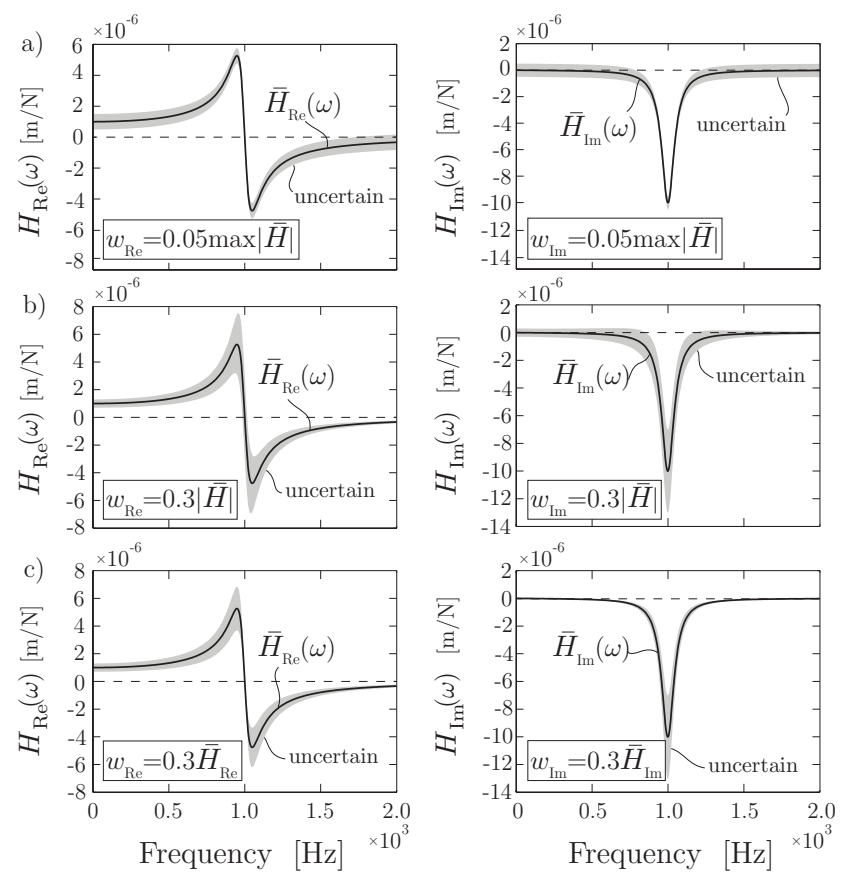

Fig. 7 FRF of a single-degree-of-freedom system in case of $5 \%$ constant frequency-independent uncertainty (a); 30\% amplitude-proportional frequency-dependent uncertainty (b); and $30 \%$ complex-proportional frequency-dependent uncertainty (c). 


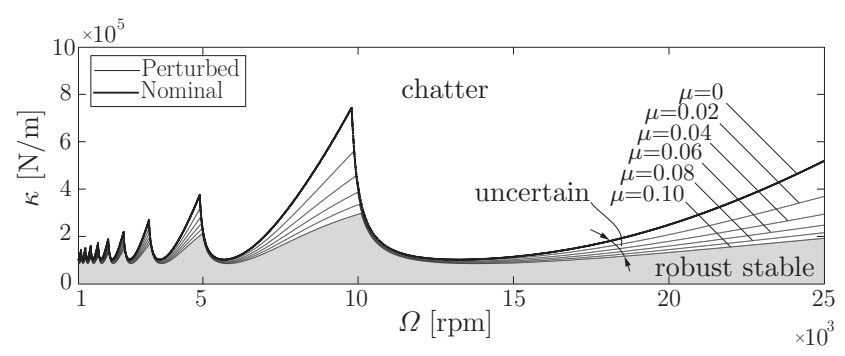

Fig. 8 Robust stability boundaries corresponding to constant uncertainty: $|\delta H(\omega)| \leq \mu \max |H(\omega)|=$ const.

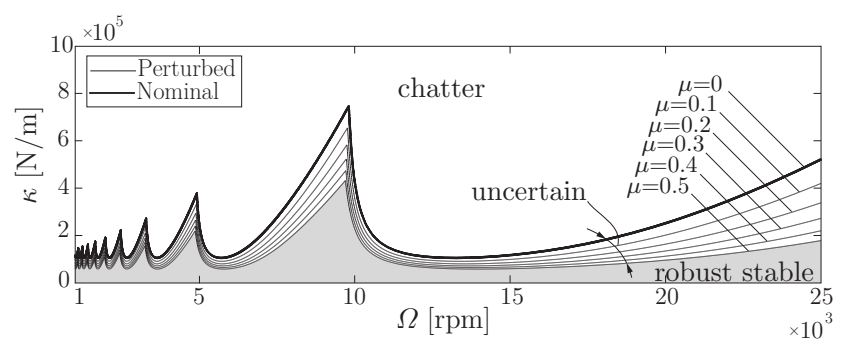

Fig. 9 Robust stability boundaries corresponding to amplitude-proportional uncertainty: $|\delta H(\omega)| \leq \mu|H(\omega)|$.

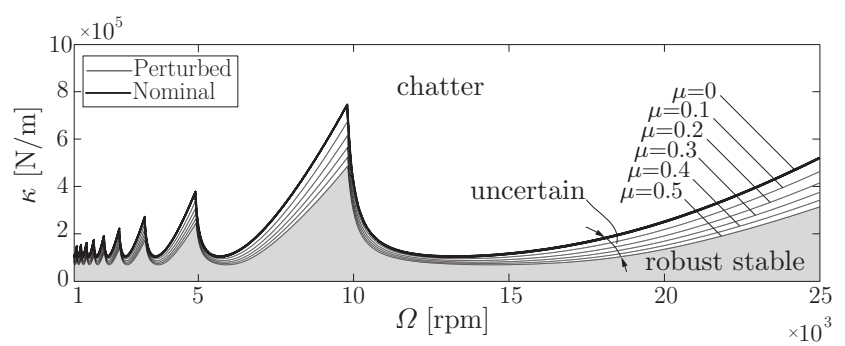

Fig. 10 Robust stability boundaries corresponding to complex-proportional uncertainty: $\left|\delta H_{\operatorname{Re}}(\omega)\right| \leq \mu\left|\bar{H}_{\operatorname{Re}}(\omega)\right|$ and $\left|\delta H_{\operatorname{Im}}(\omega)\right| \leq \mu\left|\bar{H}_{\operatorname{Im}}(\omega)\right|$.

\subsection{Analysis of a single-degree-of-freedom system}

The frequency response function of a single-degree-offreedom system is presented in Fig. 7 for different types of uncertainties. In panel a) the uncertainty is constant independently of the frequency, i.e. $|\delta H(\omega)| \leq$ $\mu \max (|\bar{H}(\omega)|)=$ const. In panel b) the uncertainty is proportional to the amplitude, i.e., $|\delta H(\omega)| \leq \mu|\bar{H}(\omega)|$. In panel c), the uncertainty is proportional to the complex function, i.e., $\left|\delta H_{\operatorname{Re}}(\omega)\right| \leq \mu\left|\bar{H}_{\operatorname{Re}}(\omega)\right|$ and $\left|\delta H_{\operatorname{Im}}(\omega)\right| \leq \mu\left|\bar{H}_{\operatorname{Im}}(\omega)\right|$. For instance, $\mu=0.1$ corresponds to maximum $10 \%$ uncertainty in any of the above types. The corresponding robust stability charts are presented in Figs. 8-10.

In Fig. 8, constant uncertainty is applied, i.e. the same complex perturbation is added to the FRF according to Fig. 7a. It is shown that this type of uncertainty affects the stability lobes to different extent at different spindle speeds. The uncertainty region widens

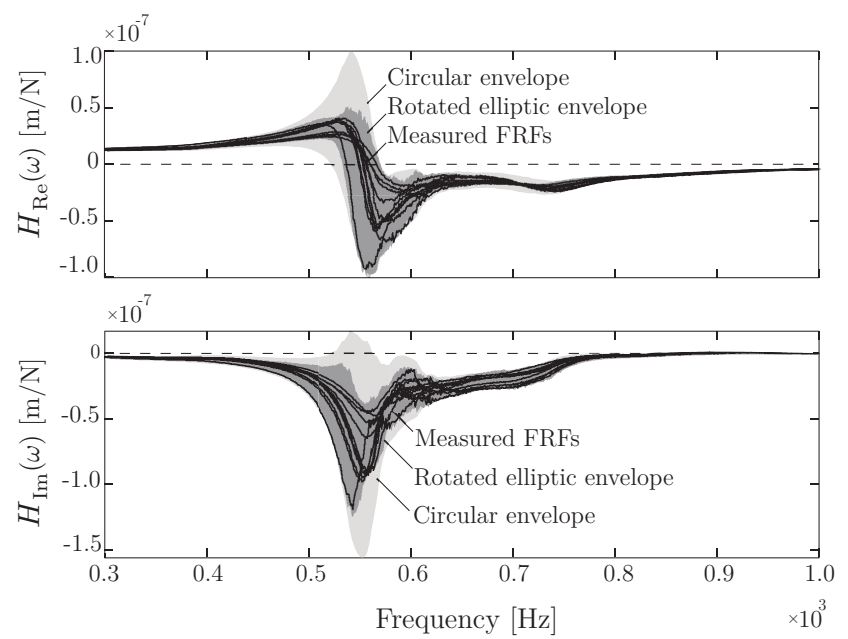

Fig. 11 Measured FRFs (solid black lines) and their circular (light gray shading) and rotated elliptic (dark gray shading) envelopes.

as one moves from the left to the right along a particular stability lobe. Already a 10\% uncertainty results in significant change in the robust stability.

The uncertainty considered in Fig. 9 is proportional to the absolute value of the FRF according to Fig. $7 \mathrm{~b}$. Again, the uncertain region increases from left to right along the stability lobes.

In Fig. 10, the applied uncertainty is proportional to the complex FRF according to Fig. 7c. The uncertain regions of the stability charts in this case are narrower compared to the previous cases.

\section{Case study}

In practical applications, the uncertainties are filtered by averaging the measured FRFs. Typically, five to ten measurements are performed and their average is used for the stability calculation [29].

In this case study, the FRF of a turning tool was measured five times, and the five FRFs were averaged in order to filter noise. Then the tool was removed, the tool holder was driven back and forth, the tool was placed back and the same FRF was measured again. This cycle was repeated ten times, which provided ten different FRFs, each being an average of five individual measurements. Figure 11 shows the ten FRFs (indicated by solid black lines) and two different uncertainty envelopes: (1) envelope with a circular cross section centered to the average of the ten FRFs such that the resulting tube comprises all FRFs (indicated by light gray shading); (2) envelope with a rotated elliptic cross section of minimum area such that the resulting tube comprises all FRFs (indicated by dark gray shading). 


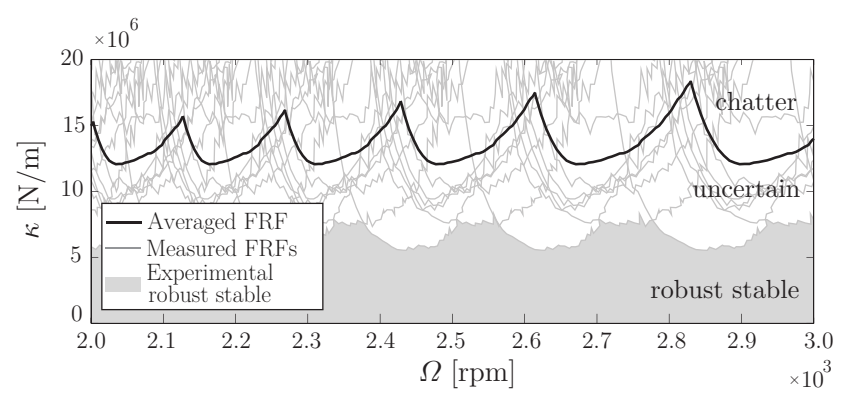

Fig. 12 Stability lobes corresponding to the averaged FRF (solid black line), the lobes corresponding to the measured FRFs (solid light gray lines), and their lower envelope called experimental robust stability (gray shading).

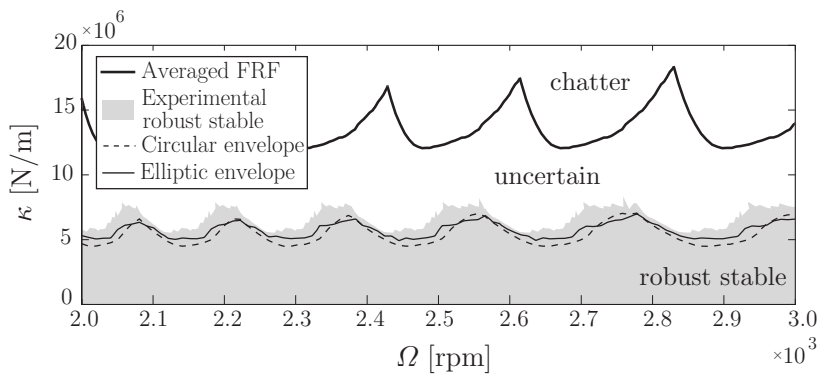

Fig. 13 Robust stability boundaries for low spindle speeds.

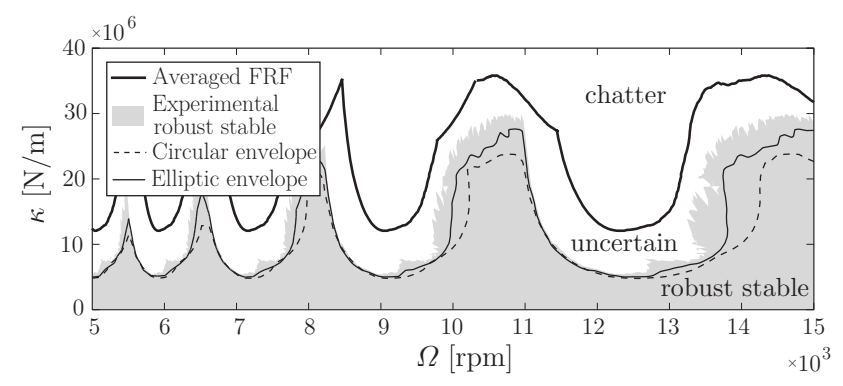

Fig. 14 Robust stability boundaries for high spindle speeds.

As can be seen, the rotated elliptic envelope gives less conservative estimation of the uncertainties.

Figure 12 shows the stability lobes corresponding to the averaged FRF and to the measured FRFs (ten times) for low spindle speeds. The lower envelope of the stability lobes corresponding to the measured individual FRFs are indicated by gray shading. This region can be called the region of experimental robust stability: within this region, the system is stable for all the ten FRFs. It can be seen that the averaged FRF strongly overestimates the robustly stable region.

Figure 13 shows the same averaged stability boundaries and the experimental robust stability boundaries together with the estimated robust stability boundaries obtained by the circular and the rotated elliptic concepts. It can be seen that both the circular and the elliptic envelopes provide almost the same curve as the experimental robust stability boundary. Since the fit- ted tubes comprises the measured FRFs, the estimation of the robust boundary is always conservative, i.e., the circular and the elliptic estimations are below the experimental robust stability boundary.

In Fig. 14, the robust stability boundaries are shown for high spindle speeds. In this case, the structure of the robust stability boundaries are more segmented than in the low spindle speed region. The robust stability estimations still provide a good approximation of the lower envelope.

\section{Conclusion}

Prediction of the stability of machining operations always involves several sources of uncertainties. Dynamic behavior of the machine-tool-workpiece system is a typical uncertain component, which is usually overcome by averaging a series of FRFs. Fitting of modal parameters is also a tool to handle uncertainties: it filters only the significant modes, which can clearly be distinguished from noise. However, if some modes are not identified properly, then the resulted stability diagrams may not reflect the properties of the real structure.

Robust stability estimations in terms of stability radii is a useful tool for single- or low-degree-of-freedom systems with a few well-structured uncertain parameters, but cannot be used for multiple-degrees-of-freedom systems with many different types of parameter uncertainties. It is still a useful tool to provide conservative estimations of robust stability boundaries.

In this paper, a frequency-domain method was proposed to the robust stability analysis of machining operations, which uses directly the measured FRFs without any filtering and modal parameter identification. The method is based on an envelope fitting around the measured FRFs combined with some considerations of the single-frequency method.

Application of the method to a single-degree-of-freedom model of orthogonal cutting operation showed that the robust stability boundaries can significantly be smaller than the stability boundaries corresponding to the averaged FRF.

An experimental study was also performed using ten different measured FRFs of a turning tool. The analysis demonstrated that the experimental robust stability boundary can well be approximated by the proposed method. This analysis also confirmed that the robust stability region can significantly be smaller than the stability region of the averaged model. This observation may explain the mismatch between stability lobe predictions and actual cutting tests, which is often experienced in machine tool chatter research. It was also 
shown that the robust stability boundaries are more sensitive at higher spindle speeds and at higher depths of cut, which highlights the significance of the prediction of robust stability boundaries in high performance machining.

The proposed method can be applied to time invariant cutting operations, such as turning operation or milling operation with large radial immersion and large number of cutting teeth. Generalization to time-varying systems, such as the milling operation with low radial immersions, using the concept of the multi-frequency method is not straightforward. Independent perturbations of the modulations of the FRF around the chatter frequency cannot be described properly using this approach.

\section{Acknowledgment}

This work was supported by the Hungarian National Science Foundation under grant OTKA-K105433. The research leading to these results has received funding from the European Research Council under the European Unions Seventh Framework Programme (FP/20072013) / ERC Advanced Grant Agreement n. 340889.

\section{References}

1. Altintas, Y.: Manufacturing Automation - Metal Cutting Mechanics, Machine Tool Vibrations and CNC Design, Second Edition. Cambridge University Press, Cambridge (2012)

2. Altintas, Y., Budak, E.: Analytical prediction of stability lobes in milling. CIRP AnnManuf Techn 44, 357-362 (1995)

3. Bachrathy, D., Stepan, G.: Improved prediction of stability lobes with extended multi frequency solution. CIRP AnnManuf Techn 62, 411-414 (2013)

4. Budak, E., Altintas, Y.: Analytical prediction of chatter stability in milling, part i: General formulation. J Dyn SystT ASME 120, 22-30 (1998)

5. Butcher, E.A., Bobrenkov, O.A., Bueler, E., Nindujarla, P.: Analysis of milling stability by the chebyshev collocation method: algorithm and optimal stable immersion levels. J Comput Nonlin DynT ASME 4, 031,003 (2009)

6. Ding, Y., Zhu, L.M., Zhang, X.J., Ding, H.: A fulldiscretization method for prediction of milling stability. Int J Mach Tool Manu 50, 502509 (2010)

7. Dombovari, Z., Wilson, R.E., Stepan, G.: Estimates of the bistable region in metal cutting. P Roy Soc AMath Phy 464, 3255-3271 (2008)

8. Graham, E., Mehrpouya, M., Nagamune, R., Park, S.: Robust prediction of chatter stability in micro milling comparing edge theorem and lmi. CIRP Journal of Manufacturing Science and Technology 7, 29-39 (2014)

9. Graham, E., Mehrpouya, M., Park, S.: Robust prediction of chatter stability in milling based on the analytical chatter stability. Journal of Manufacturing Processes 15, 508-517 (2013)
10. Hajdu, D., Insperger, T., Stepan, G.: The effect of nonsymmetric frf on machining: A case study. ASME IDETC (2015)

11. Hajdu, D., Insperger, T., Stepan, G.: Sensitivity of stability charts with respect to modal parameter uncertainties for turning operations. 12th IFAC Workshop on Time Delay Systems (2015)

12. Hinrichsen, D., Pritchard, A.J.: Stability radius for structured perturbations and the algebraic riccati eauation. Systems and Control Letters 8, 105-113 (1986)

13. Hu, G., Davison, E.J.: Real stability radii of linear timeinvariant time-delay systems. Systems and Control Letters 50, 209-219 (2003)

14. Insperger, T., Stepan, G.: Semi-discretization method for delayed systems. Int J Numer Meth Eng 55, 503-518 (2002)

15. Insperger, T., Stepan, G.: Semi-discretization for timedelay systems, vol. 178. Springer, New York (2011)

16. Insperger, T., Stepan, G., Turi, J.: State-dependent delay in regenerative turning processes. Nonlinear Dynam 47, 275-283 (2007)

17. Khasawneh, F.A., Mann, B.P.: A spectral element approach for the stability of delay systems. Int $\mathrm{J}$ Numer Meth Eng 87, 566952 (2011)

18. Kienzle, O.: Spezifische schnittkrfte bei der metallbearbeitung. Werkstattstechnik und Maschinenbau 47, 224225 (1957)

19. Michiels, W., Green, K., Wagenknecht, T., Niculescu, S.I.: Pseudospectra and stability radii for analytic matrix functions with application to time-delay systems. Linear Algebra and its Applications 418, 315-335 (2006)

20. Michiels, W., Niculescu, S.I.: Stability and Stabilization of Time-Delay Systems. Society for Industrial and Applied Mathematics (2007)

21. Michiels, W., Roose, D.: An eigenvalue based approach for the robust stabilization of linear time-delay systems. Int. J. Control 76(7), 678-686 (2003)

22. Munoa, J., Dombovari, Z., Mancisidor, I., Yang, Y., Zatarain, M.: Interaction between multiple modes in milling processes. Mach Sci Technol 17, 165-180 (2013)

23. Park, S., Qin, Y.: Robust regenerative chatter stability in machine tools. Int J Adv Manuf Technol 33, 389402 (2007)

24. Qiu, L., Bernhardsson, B., Rantzer, A., Davison, E.J., Young, P.M., Doyle, J.C.: A formula for computation of the real stability radius. Automatica 31(6), 879-890 (1995)

25. Richardson, M.H., Formenti, D.L.: Parameter estimation from frequency response measurement using rational fraction polinomials. In: 1st IMAC Conference. Orlando, Florida (1982)

26. Stepan, G.: Retarded dynamical systems. Longman, Harlow (1989)

27. Stepan, G., Dombovari, Z., Munoa, J.: Identification of cutting force characteristics based on chatter experiments. CIRP AnnManuf Techn 60, 113-116 (2011)

28. Stépán, G., Kalmár-Nagy, T.: Nonlinear regenerative machine tool vibrations. In: Proceedings of DETC'97, ASME Design and Technical Conferences, pp. 1-11. Sacramento, CA, USA (1997)

29. Stepan, G., Munoa, J., Insperger, T., Surico, M., Bachrathy, D., Dombovari, Z.: Cylindrical milling tools: comparative real case study for process stability. CIRP AnnManuf Techn 63(1), 385-388 (2014)

30. Tlusty, J., Spacek, L.: Self-excited vibrations on machine tools. Nakl. CSAV, Prague. in Czech. (1954) 
31. Tobias, S.: Machine-tool Vibration. Blackie, Glasgow (1965)

32. Totis, G., Albertelli, P., Sortino, M., Monno, M.: Efficient evaluation of process stability in milling with spindle speed variation by using the chebyshev collocation method. J Sound Vib 333, 646668 (2014)

33. Trefethen, L.N., Embree, M.: Spectra and Pseudospectra. Princeton Univ. Press (2005)

34. Verboven, P.: Frequency-domain system identification for modal analysis, PhD Thesis. Vrije Universiteit Brussel, Pleinlaan 2, B-1050 Brussels, Belgium (2002)

35. Wagenknecht, T., Michiels, W., Green, K.: Structured pseudospectra for nonlinear eigenvalue problems. Journal of Computational and Applied Mathematics 212, 245259 (2008)

36. Zatarain, M., Dombovari, Z.: Stability analysis of milling with irregular pitch tools by the implicit subspace iteration method. Int J Dynam Control 2, 26-34 (2014)

37. Zhang, X.J., Xiong, C.H., Ding, Y., Feng, M.J., Xiong, Y.L.: Milling stability analysis with simultaneously considering the structural mode coupling effect and regenarative effect. Int J Mach Tool Manu 53, 127-140 (2012) 